\title{
THE SMITHSONIAN ASTROPHYSICAL OBSERVATORY SUBMILLIMETER WAVELENGTH ARRAY
}

\author{
COLIN R. MASSON \\ Center for Astrophysics, 60 Garden St., Cambridge, MA 02138.
}

\begin{abstract}
The Smithsonian Astrophysical Observatory (SAO) is building a submillimeter-wavelength interferometer array. The telescope will be located at a high mountain site and will operate between 200 and $900 \mathrm{GHz}$. The array will consist of six movable $6 \mathrm{~m}$ antennas, on a $Y$ shaped track, with maximum baselines of $500 \mathrm{~m}$, giving sub-arcsecond resolution. It will operate simultaneously in 2 different bands, and will have a versatile spectrometer.
\end{abstract}

\section{INTRODUCTION}

The submillimeterwave interferometer array (SMA) project was initiated in 1983 when a committee was set up at SAO to study the prospects for submillimeter interferometry (Moran et al. 1984). In 1986, the first funding was secured to establish a receiver laboratory at SAO. The design study was initiated in 1988, and construction funding started in 1990 (FY 1991), with first light expected in 1996. The estimated cost of the project is $\$ 35$ million.

TABLE I Array Characteristics

\begin{tabular}{lll} 
Number of antennas & 6 & \\
Diameter of antennas & $6 \mathrm{~m}$ & \\
Antenna surface accuracy & $15 \mu \mathrm{m} \mathrm{rms}$ & \\
Baseline configuration & $\mathrm{Y}$ & \\
Maximum baseline & $500 \mathrm{~m}$ & \\
Operating frequency & $230 \mathrm{GHz}$ & $-860 \mathrm{GHz}$ \\
Maximum resolution & 0 . $^{\prime \prime}$ & $-00^{\prime \prime}$ \\
Field of view & $54^{\prime \prime}$ & $-15^{\prime \prime}$ \\
Number of simultaneous $\mathrm{rX}$ & 2 & \\
Bandwidth per receiver & $2 \mathrm{GHz}$ & $\left(700 \mathrm{~km} \mathrm{~s} \mathrm{~s}^{-1}\right.$ at $\left.860 \mathrm{GHz}\right)$ \\
Correlator & $\mathrm{XF} \mathrm{Digital}$ & \\
Channels per receiver & 2048 & \\
\hline
\end{tabular}


Table 1 gives a brief overview of the planned array. The array will be expandable, so many of the characteristics, such as i.f. bandwidth and number of simultaneous receivers may be increased later. A summary of the design status in mid-1990 was presented by Masson (1990a).

\section{STRUCTURE AND SITING OF THE ARRAY}

The $6 \mathrm{~m}$ diameter of the antennas is set by the smallest size consistent with the large and expensive receiver package, and by the sensitivity required for phase calibration. With 6 antennas, 15 baselines are available simultaneously, permitting reasonable maps to be made in a single transit of a source. The range of baseline length available with one $Y$ configuration of 6 antennas is roughly a factor of 10 . The antennas must be movable to cover baselines ranging from $6 \mathrm{~m}$ to $500 \mathrm{~m}$.

The choice of site for the array will be made in early 1991. It is dominated by the need to minimize atmospheric water vapor, which has $\tau \sim 1$ at $860 \mathrm{GHz}$ for $1 \mathrm{~mm}$ of precipitable water vapor, so the array will be located at a high mountain site. A considerable amount of water vapor data has been collected by the University of Arizona on Mount Graham. SAO joined with NRAO in 1989 to install a $225 \mathrm{GHz}$ radiometer to test Mauna Kea. Based on the preliminary results, the median opacity on Mauna Kea is 0.1 , and for $10 \%$ of the time the opacity is below 0.05 . The opacity in the high frequency windows is approximately 20 times greater than at $225 \mathrm{GHz}$.

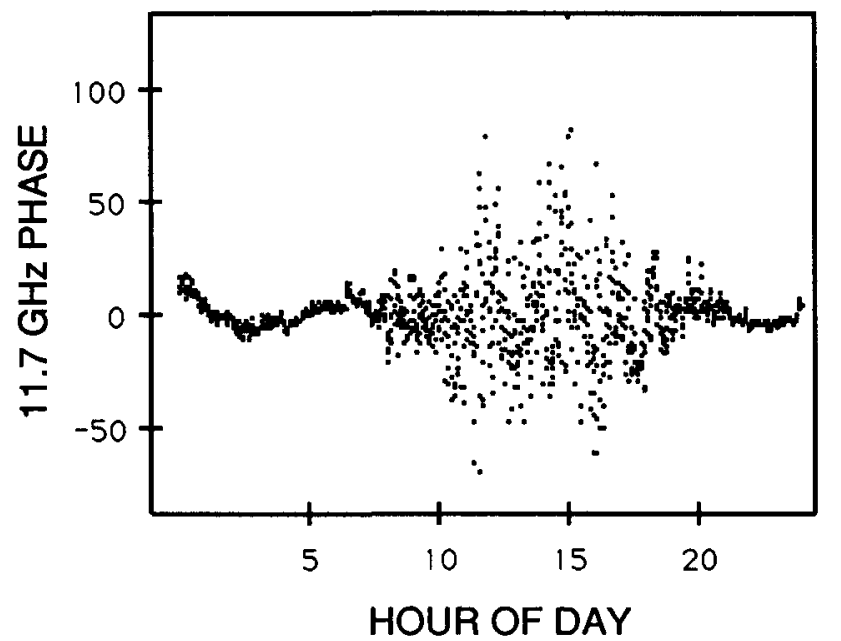

Fig. 1. 1 minute averages of the $11.7 \mathrm{GHz}$ phase during one day $(9 / 25 / 90)$ on Mauna Kea, after removing satellite motion. 
A second important site characteristic is the stability of the atmosphere. To characterize this, both for the purpose of site comparison and for design of the optimum array, SAO has constructed a radio phase monitor (Masson 1990b), which was installed at Mauna Kea in September 1990. The device is a small interferometer, with a baseline of $100 \mathrm{~m}$, which measures a beacon tone from a geostationary satellite, at a frequency of $11.7 \mathrm{GHz}$. Although this is much lower than the operating frequency of the SMA, the measurements can easily be extrapolated. Figure 1 shows the output for one day which had slightly worse than average weather. However, even in the best weather $\left(\tau_{225} \sim 0.03\right)$ there is some degradation of the phase during the daytime.

The daytime phase variations require extremely large modulations of humidity. In the daytime, the peak phase excursions of $\sim 100^{\circ}$ correspond to a excess path length of $80 \mathrm{~m}$ with a relative humidity of $100 \%$ in front of one of the antennas. Since the antennas are only $100 \mathrm{~m}$ apart, and the water is concentrated in the lower $2 \mathrm{~km}$ of the atmosphere, this suggests that the phase fluctuations occur at a boundary between very wet and very dry air. On the other hand, the lowest phase variations observed during the first month were $\sim 0.4^{\circ} \mathrm{rms}$ at $11.7 \mathrm{GHz}$, which would give a synthesized beam of 0.25 at submillimeter wavelengths. Baselines of several hundred meters would be coherent at the lower operating frequencies of the SMA.

\section{HARDWARE}

The antennas represent the largest single cost of the project. Developments for IRAM and the SMT antennas have shown that sufficiently accurate $(15 \mu \mathrm{m}$ surface) antennas can be made from carbon-fiber-reinforced plastic (cfrp), but the use of metal panels has not yet been ruled out for our rather small antennas. The backup structure will probably be made using cfrp to ensure stable pointing and phase. It is expected that the antennas will operate out in the open, since radomes or astrodomes would be difficult to transport and radomes would be lossy. The initial studies will be completed by the first quarter of 1991, when a formal request for quotations will be issued for the final design and the construction of the first antennas.

The largest technical challenge is presented by the receivers. In the upper octave ( $\geq 500 \mathrm{GHz}$ ), suitable receivers have not yet been built, but up to $500 \mathrm{GHz}$, current technology can provide adequate receivers. At these lower frequencies, SIS receivers in conjunction with multiplied Gunn local oscillators give the best performance, and superconducting mixers are therefore intended for all frequencies of the array. It is expected that future development of local oscillator and superconducting mixer technology will extend the range of these receivers to cover the needs of the SMA at all frequencies. A receiver laboratory was therefore set up in 1987 as the first part of the SAO submillimeter effort. A waveguide receiver has been constructed for $230 \mathrm{GHz}$ and experiments are being carried out with various concepts for high-frequency receivers. 
The distribution of stable local oscillator signals is harder than in existing interferometers, because of the high operating frequency, but there are no fundamental obstacles. Transmission of wideband I.F. signals ( $\geq 4 \mathrm{GHz}$ per antenna) may require the use of optical fibers. The transmission of local oscillator reference signals over fiber will be explored, but conventional cable is acceptable for the short distances involved.

The correlator will use conventional digital technology to cover a bandwidth of $4 \mathrm{GHz}$ ( $2 \mathrm{GHz}$ per receiver). The XF technique has been chosen over the FX, because it is more economical for the number of antennas and bandwidths involved in the SMA and because it offers greater flexibility. The correlator which was devised in a study by Haystack Observatory (Levine and Rogers 1989 ) breaks the $4 \mathrm{GHz}$ band up into $32 \mathrm{MHz}$ segments, each of which is digitized and processed into 32 complex channels. In full (four-quadrant) polarization mode, both receivers operate at the same frequency and 1024 channels are available across the $2 \mathrm{GHz}$ band. In non-polarization mode, 2048 channels cover the $4 \mathrm{GHz}$ band at low resolution ( $2 \mathrm{MHz}$ ) and a further 2048 channels are available for distribution across parts of the band to increase the spectral resolution.

\section{CALIBRATION AND DATA REDUCTION}

Amplitude calibration of the array will be carried out by conventional 'chopper-cal' methods. Phase calibration presents more of a problem, particularly at the highest frequencies, where the system sensitivity will be fairly low. During high-frequency operation, a second receiver will operate simultaneously at lower frequencies $(\leq 345 \mathrm{GHz})$ for phase calibration.

The restricted field of view may be a significant limitation for some sources, particularly at the highest frequencies. Extensive use of mosaicing will probably be necessary and the individual antennas will be equipped for operation in single-dish mode, to gather the necessary low-resolution data.

It is planned that data calibration and preliminary imaging will be performed nearly in real time for monitoring the progress of the observations. The offline demand for calibration, imaging and analysis will be handled by a minisupercomputer or workstations. The array will be remotely controlled, initially from a local base, but eventually from Cambridge. This will permit the flexible scheduling necessary to optimise use of the best weather conditions.

\section{REFERENCES}

Levine, J. I., and Rogers, A. 1989. SMA Technical Report No. 11. Masson, C. R. 1990a. Proc. 29th Liege Intl. Astrophys. Coll., ESA, in press. Masson, C. R. 1990b. SMA Technical Report No. 30. Moran, J., Elvis, M. S., Fazio, G. G., Ho, P. T. P., Myers, P. C., Reid, M. J., and Willner, S. P. 1984, A Submillimeter-elength Telescope Array: Scientific, Technical, and Strategic Issues, SAO, Cambridge MA. 
Greg Hennessy: If Mt. Graham is unavailable due to environmental concerns, are any other sites in the continental United States under consideration.

Colin Masson: No

Ray Norris: How bad was the weather when you obtained the 50 degree rms phase noise?

Colin Masson: This was at midday on Mauna Kea when there were probably cumulus clouds above the mountain top. 